\title{
Benefícios de técnicas cognitivo- comportamentais em terapia de grupo para o uso indevido de álcool e drogas
}

\author{
Benefits of cognitive behavior techniques in group \\ therapy for alcohol and drug abuse \\ Ana Carolina Robbe Mathias', Marcelo Santos Cruz²
}

\section{RESUMO}

A entrevista motivacional e a prevenção de recaída são abordagens de tratamento para pessoas com problemas relativos ao uso indevido de álcool e drogas. Neste trabalho,

Palavras-chaves

Abuso de álcool,

abuso de drogas,

motivação, recaída.

\section{Key-words}

Alcohol abuse, drug abuse, motivation, relapse. apresentamos o caso de um paciente demonstrando a utilização das duas abordagens associadas em tratamento em grupo e descrevemos o uso das técnicas, as várias etapas do tratamento e os resultados alcançados. São discutidos os resultados encontrados e as vantagens das técnicas.

\section{ABSTRACT}

Motivational Interviewing and relapse prevention are treatment approaches for individuals with alcohol or drug abuse problems. This article describes a group therapy treatment case, showing the association of both techniques. Each step of the treatment techniques is demonstrated and exemplified as long as their results. Results and advantages of the techniques are discussed.

\section{INTRODUÇÃO}

Em virtude da relevância dos problemas relacionados ao uso de álcool e outras drogas, é crescente a preocupação dos profissionais de saúde com a eficácia dos tratamentos propostos. Entre as maiores dificuldades e motivos de abandono de tratamento, encontram-se a motivação para modificar ou interromper o uso dessas substâncias e as recaídas freqüentes. A prevenção da recaída (PR) (Marlatt e Gordon, 1993) e a entrevista motivacional (EM) (Miller e Rollnick,
2001) são duas abordagens psicoterápicas propostas para tratar de forma específica pessoas com esses problemas.

A PR tem como objetivo capacitar os pacientes para prever e lidar com a recaída. A técnica foi desenvolvida a partir da observação de que o reinício do uso (lapso) pode ser seguido de sua interrupção, restabelecimento e continuidade de tratamento, ou pela persistência no uso da droga, constituindo-se em recaída plena (Marlatt e Gordon, 1993). Com a técnica de prevenção da recaída, o paciente desenvolve habilidades para interromper a evolução de um lapso para uma recaída.

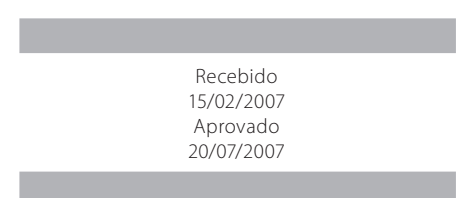

1 Universidade Federal do Rio de Janeiro (UFRJ).

2 Programa de Estudos e Assistência ao Uso Indevido de Drogas (PROJAD/IPUB/UFRJ). 
A EM tem como objetivo primordial tratar as pessoas desmotivadas, despreparadas e desencorajadas para mudar de comportamento (Miller e Rollnick, 2001). Baseia-se no modelo transteórico de Prochaska e DiClemente (1982), desenvolvido com o intuito de compreender o mecanismo que leva à mudança de um comportamento. Os autores descreveram o modelo analisando a prontidão para mudança com base em cinco estágios, que vão desde o paciente não reconhecer seu problema (pré-contemplação) até a manutenção de um novo comportamento. Nesse processo, o paciente passa a perceber o problema (contemplação), se prepara para mudar (preparação para ação) e tomar ações decisivas de mudança (ação) (Velasquez et al., 2001). Desta forma, na perspectiva da EM, a motivação não é vista como uma característica pessoal ligada à vontade própria, e sim como algo que pode ser criado e incrementado (Miller, 1996).

Tanto a PR como a EM têm como características serem estruturadas, diretivas e focais, tendo como base a teoria cognitivo-comportamental. Por serem facilmente padronizadas e esquematizadas, são utilizadas internacionalmente em ensaios clínicos, facilitando a comparação de procedimentos e estudos (Cruz et al., 2007).

Apesar de a EM e a PR serem usadas em diversos tipos de tratamento, por serem técnicas relativamente novas, poucos trabalhos na literatura vêm demonstrando sua forma de atuação, principalmente com a utilização conjunta das duas técnicas como descrevemos no presente trabalho. No Brasil, são raros os estudos encontrados. Em uma busca por artigos que tratem dessas temáticas na base SciELO, na Revista Brasileira de Psiquiatria e no Jornal Brasileiro de Psiquiatria, no período de 1990 a 2006, encontraram-se apenas cinco artigos (Castro e Passos, 2005; Andretta e Oliveira, 2005; Marques e Furtado, 2004; Jungerman e Laranjeira, 1999; Silva et al., 1995). Nestes, não há relato do uso conjunto das técnicas, e os temas são abordados em forma de revisão de literatura ou propondo intervenções breves com poucas sessões. Sendo assim, apresentamos este caso com o intuito de demonstrar praticamente o uso das duas abordagens juntas, acompanhando a motivação do sujeito em todo processo de mudança, inclusive com a descrição da sua forma de lidar com a recaída quando esta ocorreu.

\section{RELATO DE CASO}

L., 47 anos, pardo, natural do Rio de Janeiro, solteiro, sem ocupação profissional, procurou atendimento no ambulatório do Instituto de Psiquiatria da UFRJ, no Programa de Estudos e Assistência ao Uso Indevido de Drogas (PROJAD). Ele fazia uso de álcool havia pelo menos 25 anos e de cocaína havia 20 anos, e já tinha se submetido a inúmeros tratamentos, tendo sido internado por diversas vezes.
Sempre abandonou esses tratamentos depois de recaídas, a partir das quais voltava a usar as substâncias como antes. Em sua história, períodos de meses de abstinência acompanhavam-se de grande vontade de usar álcool e cocaína e de sensação de vazio relacionada à falta de ocupação e de esperança no futuro. Para adquirir a droga, L. freqüentava locais perigosos e se envolvia com traficantes, colocando-se em diversas situações de risco. L. não conseguia se estabelecer em nenhum emprego, pois, ao receber o primeiro salário, desistia de tudo e passava dias se drogando. Sua relação com a família não era boa, tendo contato com poucos familiares.

No momento em que procurou tratamento, L. estava sem consumir nenhuma substância havia uma semana, mas relatava muita vontade de usar. Notava-se grande ambivalência, pois mesmo informando que queria deixar de usar, em seu discurso percebia-se que estava procurando o tratamento mais por causa da mãe e da irmã do que por si próprio. Também relutava em abandonar o grande prazer que a droga Ihe proporcionava.

\section{Evolução clínica}

Nos primeiros meses do tratamento do paciente no grupo, realizaram-se técnicas como: "Um dia típico", "prós e contras", avaliação das expectativas em relação às drogas e identificação dos estágios de mudança para que aumentasse a consciência dos sujeitos sobre as suas problemáticas em relação às drogas. Com isso, L. pôde perceber por que buscava as drogas e a função central que ocupavam em sua vida. Pôde, então, traçar objetivos concretos e conscientes para interromper o uso. Enfocaram-se as recaídas anteriores e as situações de risco para que ele conseguisse prever e evitar uma recaída, e se esta acontecesse, conseguisse manejar para que esta fosse apenas um "lapso".

Nas festividades de final de ano, L. bebeu e usou cocaína. Como estava mais consciente de seu problema, conseguiu prever que não conseguiria parar de usar e recorreu a uma breve internação. Desta forma, L. conseguiu que esse tropeço não se transformasse numa recaída plena. A internação permitiu a L. retornar ao estágio de ação.

Com sua volta ao grupo e seguindo regularmente o tratamento, o paciente foi recuperando sua auto-estima e a confiança de sua família por meio do trabalho de valores, relacionamentos e assertividade. Para assegurar a escolha de L. de não usar drogas, realizaram-se técnicas como relaxamento, reforço das conquistas e solução de problemas.

L. começou a trabalhar informalmente e ele, que antes gastava em drogas tudo o que recebia, passou a manejar adequadamente seu dinheiro. Para maior esclarecimento das técnicas relatadas anteriormente, veja Velasquez et al. (2001). 


\section{DISCUSSÃO}

No caso descrito, observamos que o paciente chegou ao tratamento no estágio de contemplação, pois mesmo disposto a interromper o uso de cocaína e álcool, oscilava em vários momentos e ainda se sentia muito atraído pelas drogas. Os exercícios sobre os estágios de mudança demonstraram que o paciente pôde ter maior percepção do seu problema, adquirindo responsabilidade por seus atos, o que gerou maior comprometimento com o tratamento (Velasquez et al., 2001).

Com uma boa coleta de informações sobre as principais dificuldades do paciente, foi possível, durante as sessões do grupo, contemplar as suas necessidades, como as formas de recusar a droga e lidar com a fissura, a conquista de novas amizades, a confiança dos familiares e o manejo de dinheiro. Com isso, o paciente pôde desenvolver um novo comportamento menos prejudicial, podendo chegar ao estágio de manutenção.

Pode-se observar que o modo de compreensão da recaída e a forma como esta é trabalhada com os pacientes, somada ao estilo motivacional não confrontativo, podem ser fatores importantes para que os pacientes não interrompam o tratamento. Desta forma, o estilo acolhedor da EM, aliado à forma de abordagem da recaída da PR, foi efetivo para o paciente aderir ao tratamento e conseguir a abstinência. Como se verifica no trabalho de van Horn e Bux (2001), as discussões provocadas durante as sessões produziram reflexões nos dias seguintes. Não era raro o paciente relatar que o depoimento de outros pacientes o levou a modificar sua forma de pensar sobre seu problema. Isto nos mostra a importância do tratamento em grupo, que facilita a troca de experiências e levantamento de questões que não aconteceriam em um tratamento individual (Cruz et al., 1999).

O tratamento em grupo mostrou-se bastante eficiente para este paciente, facilitando seu engajamento no processo de mudança, o que confirma outros estudos sobre a efetividade da EM e PR em formato de grupo (Foote et al., 1999; Lincourt et al., 2002; van Horn e Bux, 2001).

\section{CONCLUSÕES}

No caso descrito, a EM em grupo mostrou-se bastante eficiente para motivar o paciente a interromper o uso da droga e engajar-se no tratamento. A abordagem da recaída durante as sessões também foi importante, pois, segundo o paciente, permitiu que ele tivesse coragem de retornar ao grupo e alcançar o seu objetivo.

Com isso, o serviço se torna mais diversificado e flexível, podendo contemplar um número maior de pacientes. Por fim, para que se amplie o conhecimento sobre o atendimento em grupo a partir da perspectiva da EM e PR, suas potencialidades e limites, é de extrema importância a realização de mais estudos nesta área.

\section{REFERÊNCIAS}

Andretta I, Oliveira MS. A técnica da entrevista motivacional na adolescência. Psicol Clin, 17 (2): 127-39, 2005.

Castro MMLD, Passos SRL. Entrevista motivacional e escalas de motivação para tratamento de dependência de drogas. Rev Psiq Clínica, 32 (6):330-5, 2005.

Cruz MS, Barbeito MM, Mathias ACR. 0 papel das psicoterapias na abordagem do uso abusivo de drogas. In: Gigliott A, Guimarães A (orgs.). Dependência, compulsão e impulsividade. Rio de Janeiro: Rubio p. 187-191, 2007.

Cruz MS et al. Criação de um serviço de atendimento a usuários de drogas em ambulatório do Instituto de Psiquiatria da UFRJ: experiência de dois anos. Informação Psiquiátrica, 18 17-22, 1999.

Foote J et al.. A group motivational treatment for chemical dependency. Journal of substance abuse treatment, 17 (3):181-92, 1999.

Jungerman FS, Laranjeira R. Entrevista motivacional: bases teóricas e práticas. J Bras Psiquiatr 48(5):197-207, 1999.

Lincourt P, Kuettel TJ, Bombardier CH. Motivational interviewing in a group setting with mandated clients: a pilot study. Addictive behaviors, 27:381-91, 2002.

Marlatt GA, Gordon JR. Prevenção de recaída: estratégias no tratamento de comportamentos aditivos. Porto Alegre: Artes Médicas; 1993.

Marques ACPR, Furtado EF. Intervenções breves para problemas relacionados ao álcool. Rev Bras Psiquiatr, 26 (Supl I):28-32, 2004

Miller WR. Motivational interviewing: reserch, pratice, and puzzles. Addictive Behaviours, 21 $835-42,1996$

Miller WR, Rollnick S. Entrevista motivacional: preparando as pessoas para mudança de comportamento. Porto Alegre: Artes Médicas; 2001

Prochaska J0, DiClemente CC. Transtheoretical therapy: toward a more integrative model of change. Psychotherapy: Theory, Research and Practice, 20:161-73, 1982.

Silva EA, Ferri CP, Formigoni MLOS. Situações de recaída em pacientes dependentes de álcool e outras drogas durante o tratamento: um estudo preliminar. J Bras Psiq 44(6):311-5, 1995.

van Horn DHA, Bux DA. A pilot test of motivational interviewing groups for dually diagnosed in patiens. Journal of substance abuse treatment, 20:191-5, 2001

Velasquez MM et al. Group treatment for substance abuse: a stage-of-change therapy manual. New York: The Guilford Press; 2001. 